\title{
Prevalence and Impact of Inflammatory Bowel Disease-Irritable Bowel Syndrome on Patient-reported Outcomes in CCFA Partners
}

\author{
Maisa I. Abdalla, MD, MPH, ${ }^{*}{ }^{+}$Robert S. Sandler, $M D, M P H_{,}^{*}$ Michael D. Kappelman, $M D, M P H_{,}^{*}$ \\ Christopher F. Martin, MSPH, ${ }^{*}$ Wenli Chen, MA, MS, ${ }^{*}$ Kristen Anton, MS, and Millie D. Long, MD, MPH*
}

\begin{abstract}
Background: Inflammatory bowel disease (IBD) patients with persistent symptoms despite no or minimal inflammation are frequently described as having an overlap of IBD and irritable bowel syndrome (IBD-IBS). Limited data are available on how IBS impacts the individual patient with IBD. In this study, we aimed to evaluate the prevalence of IBD-IBS and investigate its impact on patient-reported outcomes.
\end{abstract}

Method: We performed a cross-sectional analysis within the CCFA Partners Study. Bivariate analyses and logistic regression models were used to investigate associations between IBD-IBS and various demographic, disease factors, and patient-reported outcomes including anxiety, depression, sleep disturbances, pain interference, and social satisfaction.

Results: Of the 6309 participants included, a total of $1279(20 \%)$ reported a coexisting IBS diagnosis. The prevalence of IBD-IBS in this cohort was similar within disease subtypes. A diagnosis of IBD-IBS was associated with higher narcotic use compared with those with no IBS diagnosis for both Crohn's disease, $17 \%$ versus $11 \%(P<0.001)$ and ulcerative colitis/indeterminate colitis, $9 \%$ versus $5 \%(P<0.001)$. Quality of life, as measured by Short Inflammatory Bowel Disease Questionnaire (SIBDQ) was lower in patients with IBD-IBS compared with those without. IBD-IBS diagnosis was associated with anxiety, depression, fatigue, sleep disturbances, pain interference, and decreased social satisfaction.

Conclusions: In this sample of patients with IBD, high prevalence of concomitant IBS diagnosis was observed. IBD-IBS diagnosis was associated with increased narcotic use and adverse patient-reported outcome. Appropriate diagnosis, treatment, and counseling may help improve the functional status of IBD-IBS patients and decrease narcotic use.

(Inflamm Bowel Dis 2017;23:325-331)

Key Words: irritable bowel syndrome in IBD, health care utilization, patient-reported outcomes

$\mathrm{M}$ any patients with inflammatory bowel disease (IBD) continue to experience symptoms of abdominal pain and diarrhea despite minimal or no active inflammation. These patients are frequently described as having an overlap between IBD and irritable bowel syndrome (IBD-IBS). The awareness of IBD-IBS has increased over time as several studies have reported a higher prevalence of IBS among patients with IBD compared with the general population. ${ }^{1-5}$ In a meta-analysis by Halpin et al, ${ }^{6}$ pooled data from 13 studies revealed an overall

Received for publication September 16, 2016; Accepted December 1, 2016.

From the *Department of Medicine, Division of Gastroenterology and Hepatology, University of North Carolina at Chapel Hill, Chapel Hill, North Carolina; †Department of Medicine, Division of Gastroenterology and Hepatology, Loma Linda University, Loma Linda, California; ${ }^{\star}$ Department of Pediatrics. Division of Pediatric Gastroenterology, University of North Carolina, Chapel Hill, North Carolina; and ${ }^{\S}$ Geisel School of Medicine at Dartmouth, Hanover, New Hampshire.

Supported by the Crohn's and Colitis Foundation of America, Patient Centered Outcomes Research Institute, NIH P30 DK34987 and NIH 1K08DK088957-01.

M. D. Long has consulted for Abbvie, Salix, Pfizer and Theravance pharmaceuticals. M. D. Kappelman has consulted for Abbvie, Janssen, and GlaxoSmithKline. The remaining authors have no conflict of interest to disclose.

Address correspondence to: Maisa I. Abdalla, MD, MPH, Department of Medicine, Division of Gastroenterology and Hepatology, 11234 Anderson Street, MC 1556, Loma Linda, CA 92354 (e-mail: misaabdalla@gmail.com).

DOI 10.1097/MIB.0000000000001017

Published online 13 January 2017. prevalence of IBS of $35 \%$ in patients with IBD who were in apparent remission.

Less is known about the impact of IBS on the individual IBD patient's care and functional status. IBS has been linked to disproportionate excessive health care utilization with total direct and indirect annual expenditures exceeding \$20 billion. ${ }^{7,8}$ However, it remains unclear what additional health burden it may inflict on patients with IBD. In a study by Ramos-Rivers et al, ${ }^{9}$ patients with IBD who were higher users of health resources, namely telephone encounters, were more likely to have chronic abdominal pain and psychiatric comorbidities, both features frequently seen in patients with IBS. Furthermore, it is often difficult to clinically differentiate between symptoms of IBS and those of active inflammation. Hence, patients with IBD-IBS may unnecessarily undergo repeated gastrointestinal (GI) procedures and abdominal imaging, have unnecessary escalation of immunosuppressive therapies, or a delay in initiating needed therapies. IBDIBS may also impact medication utilization, particularly rates of narcotic use, which have been shown to be higher for IBD patients with concomitant IBS diagnosis compared with those without IBS in inpatient settings. ${ }^{10}$

Although both IBD and IBS have been independently linked to reduced health-related quality of life (HRQOL) measures and have been associated with several psychiatric comorbidities, such as depression, anxiety, fatigue, and sleep 
disturbances, little is known about the impact of combined IBD and IBS on patients' functional status. ${ }^{11,12}$ Few studies have consistently shown reduced HRQOL scores in patients with IBD-IBS compared with IBD patients with no concomitant IBS diagnosis. However, variable results were seen in regard to associations with anxiety and depression..$^{2-4,13-19}$ These studies included a relatively small number of patients (range: 50-276), and varied in their inclusion criteria and the instruments used to assess the different aspects of a patients' functional status. We therefore aimed to evaluate the epidemiologic and disease-related characteristics of patients with IBD-IBS within a large sample of patients with IBD. In addition, we aimed to investigate the impact of this diagnosis on various patient-reported outcomes (PROs), including measures of functional status, and specific medication utilization.

\section{MATERIALS AND METHODS}

\section{Study Design}

We performed a cross-sectional analysis of a subset of patients within the Crohn's and Colitis Foundation of America (CCFA) Partners cohort.

\section{Study Population}

CCFA Partners is a longitudinal internet-based cohort of adult patients with IBD, which has been ongoing since 2011. The development of the cohort was previously described. ${ }^{20}$ In brief, after a baseline survey, participants are invited to complete follow-up surveys every 6 months. At baseline and every follow-up interval, data on demographics, disease subtype and characteristics, medications, measures of disease activity, and various PROs are collected. All data are self-reported.

In 2014, the surveys were updated to include the following question: "After you were diagnosed with IBD has a doctor ever told you that you had Irritable Bowel Syndrome (IBS)?" with 3 possible answers: (1) Yes, (2) No, or (3) Don't know. Participants who answered this question with either yes or no were included in this analysis $(n=6309)$.

\section{Study Variables}

Disease activity was assessed using short Crohn's Disease Activity Index (sCDAI) for Crohn's disease (CD) and Simple Clinical Colitis Activity Index (SCCAI) for ulcerative colitis (UC)/ indeterminate colitis (IC). Active disease was defined as SCDAI $\geq 150$ for $\mathrm{CD}$ and SCCAI $>2$ for UC/IC. The short IBD Questionnaire (SIBDQ) (Short Inflammatory Bowel Disease Questionnaire (SIBDQ) was authored by Dr. J. Irvine, et al, was made under license from McMaster University, Hamilton, Canada) was used to assess HRQOL. For each participant, levels of anxiety, depression, fatigue, sleep disturbances, pain interference, and social satisfaction were assessed using the Patient-Reported Outcomes Measurement Information System (PROMIS). The PROMIS measures have been previously validated in general and chronically ill populations and their performance was assessed within the CCFA Partners cohort. ${ }^{11,21,22}$ In brief, participants completed 4 items for each of the PROMIS measures. The items are then calibrated using $\mathrm{T}$-score metric to provide a mean value for the measure assessed. In the general US population, the mean for each domain is 50 with a SD of 10. A priori thresholds of $\geq 52$ (anxiety, depression, fatigue, pain interference, and sleep disturbances) and $\leq 48$ (social satisfaction) were selected to define the presence of adverse PRO within each measure. We have chosen these threshold points to allow for a clinically meaningful separation from the general population. Minimal important differences in PROMIS measures for patients with IBD have not been defined. However, research in patients with cancer, patients with chronic pain, and healthy pediatric population revealed a range of minimal important differences of 2 to 6 for several of the PROMIS measures. ${ }^{23-25}$ Numbers of clinic visits to the primary care physician (PCP) and GI physician within the previous year were used as indicators for health care utilization. High utilization was defined by $\geq 5$ visits to the PCP or GI physician within the previous year.

\section{Statistical Analysis}

We first performed bivariate analyses to evaluate associations between IBD-IBS and the various demographic and diseaserelated variables stratified by disease subtypes. Continuous variables were presented as means and SD, whereas the categorical variables were reported as percentages. Comparisons were made using Pearson's chi-square test for categorical variables and Student's $t$ test or Mann-Whitney test for continuous variables. Logistic regression models were then used to investigate independent associations between IBD-IBS and each of the 6 PROs while controlling for participants' age, body mass index, disease subtype, disease duration, sex, narcotics use, smoking status, and IBD therapies. All statistical analyses were performed using SPSS version 22.0 (IBM Corp., Armonk, NY).

\section{Ethical Considerations}

The study protocol was approved by the Institutional Review Board at the University of North Carolina.

\section{RESULTS}

\section{Study Population}

A total of 6309 participants met the inclusion criteria for the study. Of these, 3947 reported having CD and 2362 reported UC or IC. Most participants were women (71.9\%) and whites (94.1\%). The mean age of the study cohort was 44 years, and the average time between the initial diagnosis and the time of the survey completion was 14 years.

A total of $1279(20.3 \%)$ participants reported a coexisting IBS diagnosis. The proportion of patients reporting concomitant IBS diagnosis was similar among patients with CD (20.0\%) and UC/IC (20.7\%) $P=0.49$. In both CD and UC/IC groups, patients with IBD-IBS were more likely to be women and have higher body mass index and were less likely to have graduated college, as compared with those with no concomitant IBS diagnosis (Table 1). In UC/IC group, the mean age for patients with 
TABLE 1. Demographic and Disease-related Characteristics of Patients with IBD Stratified by Disease Subtype and Concomitant IBS Diagnosis

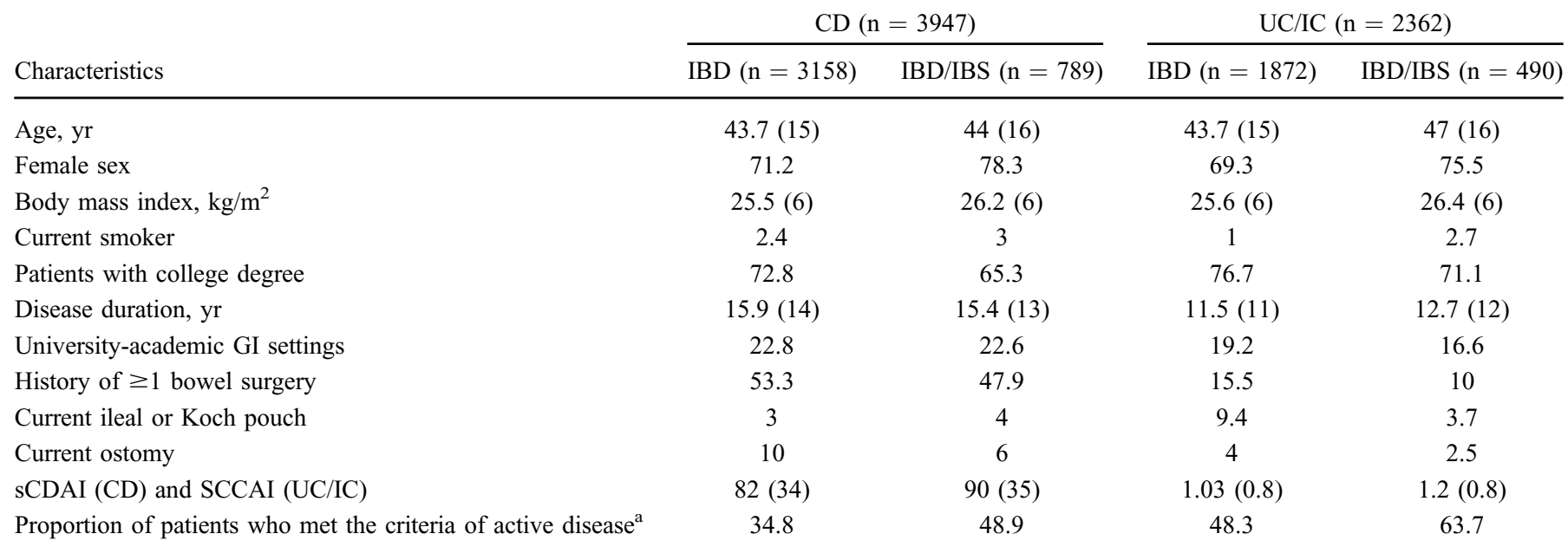

Data presented as mean (SD) and percentages.

${ }^{\mathrm{a}}$ Active disease defined as $\mathrm{SCDAI} \geq 150$ for $\mathrm{CD}$ and SCCAI $>2$ for UC/IC.

IBD-IBS was significantly higher compared with those with no IBS diagnosis (47 versus 43, respectively, $P=<0.001$ ). No significant age differences were noted between the 2 groups in patients with $\mathrm{CD}$. The proportion of patients with at least one previous abdominal surgery was lower in patients with IBD-IBS compared with those with no IBS diagnosis in both CD and UC/ IC participants. Patients with IBD-IBS had lower rates of current ileal/Kock pouch and current ostomy compared with those with no IBS diagnosis in UC/IC and CD groups, respectively. In both $\mathrm{CD}$ and UC/IC groups, patients with IBD-IBS were more likely to have a sCDAI score $\geq 150$ (CD) or SCCAI $>2$ (UC/IC) compared with those with no concomitant IBS (48.9\% versus $34.8 \%$ [CD] and $63.7 \%$ versus $48.3 \%$ [UC/IC]).

\section{Health Resources and Medications Utilization}

In both $\mathrm{CD}$ and $\mathrm{UC} / \mathrm{IC}$, patients with concomitant IBS diagnosis were more likely to report $\geq 5$ visits to their PCP and GI physician within the previous year compared with those with no concomitant IBS diagnosis (Table 2). Furthermore, using logistic regression models to assess the impact of IBD-IBS diagnosis on the likelihood that participants would report $\geq 5$ visits to their PCP and GI physicians within the previous year while controlling for age, sex, disease subtype, current narcotics use, and current use of various IBD therapies, IBD-IBS was an independent predictor for both outcomes (odds ratio [OR] 1.76, 95\% confidence interval [CI], 1.45-2.14 for PCP visits and for GI visits, OR 1.36, 95\% CI, 1.13-1.64.

Participants with IBD-IBS diagnosis were more likely to report current narcotic use compared with those with no IBS diagnosis, in both disease subtypes (Fig. 1). Among patients in clinical remission (defined by sCDAI score $<150$ for $\mathrm{CD}$ and $\mathrm{SCCAI} \leq 2$ for $\mathrm{UC} / \mathrm{IC}$ ), higher rates of narcotic use among patients with IBD-IBS were still observed in both CD (7.2\%) and UC/IC (4.7\%). No significant differences were seen in regard to IBD therapies (5-aminosalicylates, immunomodulators, and biologics) utilization between patients with IBD-IBS and those with no concomitant IBS diagnosis in both disease subtypes. In addition, IBD-IBS diagnosis was not associated with higher rates of current corticosteroids use (Table 2).

\section{Impact on Quality of Life and PROs}

On bivariate analyses stratified by disease activity, patients with IBD-IBS had lower SIBDQ scores compared with those with no concomitant IBS diagnosis. The difference was significant for both disease subtypes (Table 3). Furthermore, patients with IBD-IBS had significantly higher mean values for anxiety, depression, fatigue, pain interference, and sleep disturbances and lower social satisfaction means compared with those with no concomitant IBS diagnosis in both disease subtypes (Table 3). Using logistic regression models to investigate independent associations between IBD-IBS and the presence of each of the 6 PROs, IBD-IBS was an independent predictor for anxiety (OR 1.62, 95\% CI, 1.41-1.87), depression (OR 1.59, 95\% CI, 1.39-1.83), fatigue (OR 1.56, 95\% CI, 1.35-1.8), pain interference (OR 1.84, 95\% CI, 1.59-2.12), sleep disturbances (OR 1.61, 95\% CI, 1.4-1.86), and decreased social satisfaction (OR 1.48, 95\% CI, 1.29-1.70). Analyses were repeated within strata of disease activity, with similar results (Fig. 2).

\section{DISCUSSION}

In this large sample of self-reported patients with IBD, one in every 5 participants had a concomitant IBS diagnosis. After sex 
TABLE 2. Comparison of the Health Resources and Medication Utilization Between IBD Patients with and Without Concomitant IBS Diagnosis Stratified by Disease Subtype

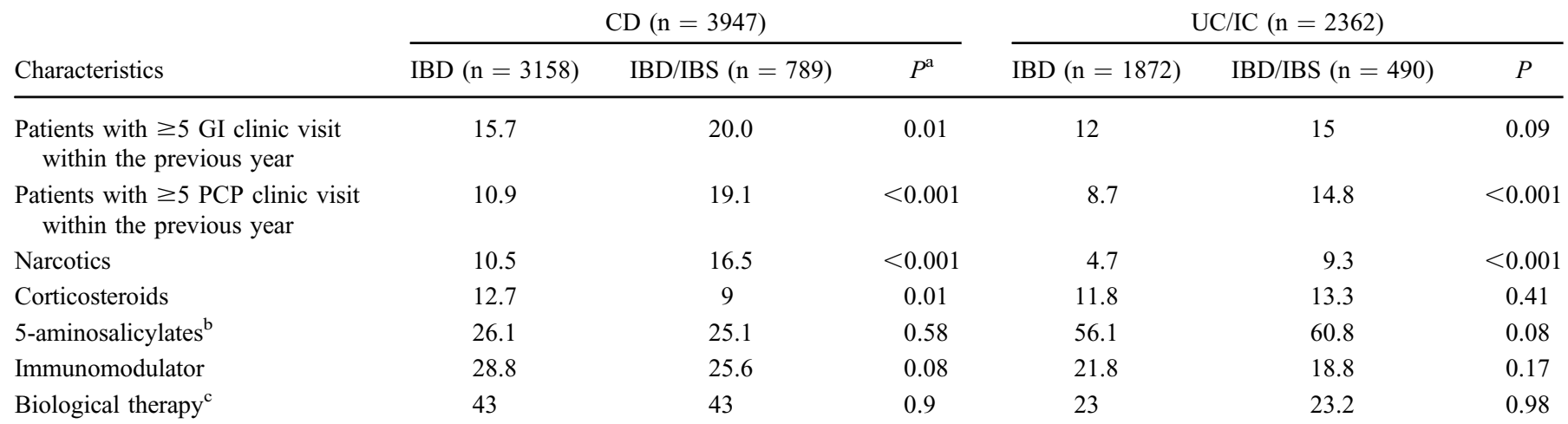

Data presented are percentages.

${ }^{\text {a} C}$ Comparisons were made using Pearson's chi-square test for categorical variables.

${ }^{\mathrm{b}}$ Azathioprine, 6-mercaptopurine, and methotrexate.

${ }^{\mathrm{c} A n t i-t u m o r}$ necrosis factor agents and antiadhesions (vedolizumab and natalizumab).

adjustment, the IBS prevalence remained almost $75 \%$ higher than the prevalence in the general US population, $19 \%$ versus $11 \%$, respectively. Contrary to the findings from several previous studies, which suggested higher IBS prevalence in CD compared with patients with UC, in our study, the prevalence was comparable within the 2 disease subtypes. , $^{2,13,14,26,27}$ The different epidemiologic distribution in our study may be related to the distinct characteristics of the studied population. Our cohort is

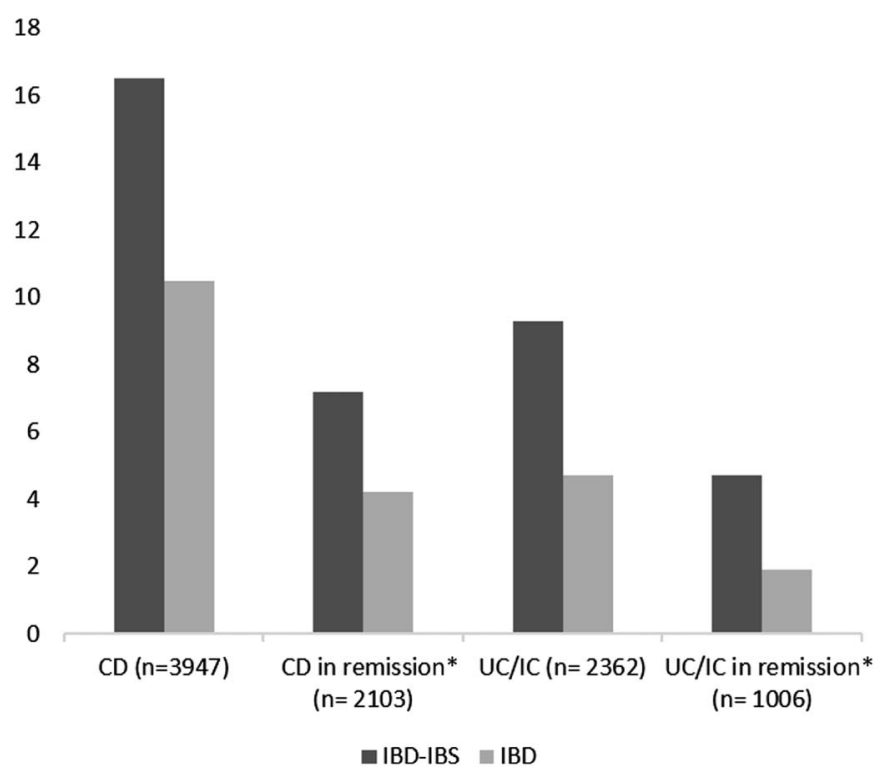

FIGURE 1. Rates of narcotics utilization among IBD patients with and without concomitant IBS diagnosis stratified by disease subtype and disease activity. ${ }^{*}$ Clinical remission defined as SCDAI $<150$ for $\mathrm{CD}$ and $\mathrm{SCCAI} \leq 2$ for UC/IC. internet based, with predominantly white females and may not represent the general IBD population. Alternatively, the small sample size in the previous studies may have influenced their reported results. Regardless of the exact prevalence in each disease subtype, it remains evident that patients with IBD have considerably higher prevalence of IBS compared with the general population.

Several other important findings were observed in this study. First, patients with IBD-IBS were more likely to have $\geq 5$ annual office visits to their PCP and GI physicians compared with those with no concomitant IBS diagnosis. This is consistent with the pattern of resource utilization in the general IBS population as IBS is estimated to be responsible $\sim 3.6$ million physician visits in the Unites States annually. ${ }^{28}$ Although clinic visits represent only one indicator of the disease burden, those visits frequently generate a trail of health expenditures related to medication prescriptions and diagnostic procedures/tests. Furthermore, it has been previously shown that patients tend to underestimate the number of physician office visits, ${ }^{29,30}$ hence, the burden may be even higher.

The second important observation in this study is the noted high rate of narcotic utilization in association with IBD-IBS. This is of particular significance for patients with IBD as the use of narcotic-based analgesia has been linked to a number of adverse effects, including higher rate of infectious complications, surgical complications, and even mortality. ${ }^{31-33}$ In a study by Long et $\mathrm{al}^{10}$ assessing risks of narcotic use in hospitalized patients with IBD, all patients with previous IBD-IBS diagnosis received narcoticbased analgesia during their hospitalization. Having CD, disease duration, previous psychiatric comorbidities, outpatient narcotic use, current smoking, and previous IBD-specific surgery were also associated with narcotic use in hospitalized patients with 
TABLE 3. Quality of Life Measures and PROs in IBD Patients with and Without IBS Diagnosis Stratified by Disease Subtype

\begin{tabular}{|c|c|c|c|c|}
\hline \multirow[b]{2}{*}{ Outcome } & \multicolumn{2}{|c|}{$\mathrm{CD}(\mathrm{n}=3947)$} & \multicolumn{2}{|c|}{$\mathrm{UC} / \mathrm{IC}(\mathrm{n}=2362)$} \\
\hline & $\operatorname{IBD}(\mathrm{n}=3158)$ & $\mathrm{IBD} / \mathrm{IBS}^{\mathrm{a}}(\mathrm{n}=789)$ & $\operatorname{IBD}(\mathrm{n}=1872)$ & $\mathrm{IBD} / \mathrm{IBS}^{\mathrm{a}}(\mathrm{n}=490)$ \\
\hline SIBDQ $^{\mathrm{b}}$ & $5(1)$ & $4(1)$ & $5.2(1)$ & $4.7(1)$ \\
\hline Depression & $50.3(9)$ & $52.7(10)$ & $49.4(9)$ & $52.2(10)$ \\
\hline Fatigue & $54.7(11)$ & $57.6(11)$ & $52.4(11)$ & $55.8(11)$ \\
\hline Social satisfaction & $49.3(10)$ & $47.1(10)$ & $50.4(10)$ & $47.2(10)$ \\
\hline
\end{tabular}

Data presented as mean (SD).

${ }^{a}$ For every evaluated outcome, the mean value was significantly different for IBD-IBS compared with IBD with no IBS diagnosis. $P$ value $<0.001$ (Student's $t$ test)

${ }^{\mathrm{b}} \mathrm{SIBDQ}$, short IBD questionnaire.

IBD. In the outpatient setting, several factors were linked to increased narcotic use in patients with IBD, including psychiatric comorbidities, female sex, history of abuse, and clinical disease activity. ${ }^{34-36}$ However, the impact of IBD-IBS on outpatient narcotic use has not been previously assessed. In our study, patients with IBD-IBS had higher rates of narcotic use compared with those with no IBS diagnosis. The differences in narcotic use remained significant even after we excluded those who met clinical criteria of active disease. Lastly, consistent with the previous reports, IBD-IBS in our cohort was associated with reduced HRQOL as measured by SIBDQ in both disease subtypes. Moreover, IBD-IBS diagnosis was found to be an independent predictor for all 6 PROs; anxiety, depression, fatigue, pain interference, sleep disturbances, and reduced social satisfaction. Those effects remain significant after we excluded patients who met clinical criteria for active disease.

There are several strengths to this study. It is the largest study to date that examines the prevalence and impacts of IBS in patients with IBD. The large sample size allowed for data stratification and separate analyses within disease subtypes. It also allowed for adequate statistical power and meaningful assessments of the different associations between IBD-IBS and PROs while taking into account many of the confounding factors. The use of validated scales for the various PROs, HRQOL, and self-reported measurement of disease activity is another strength.

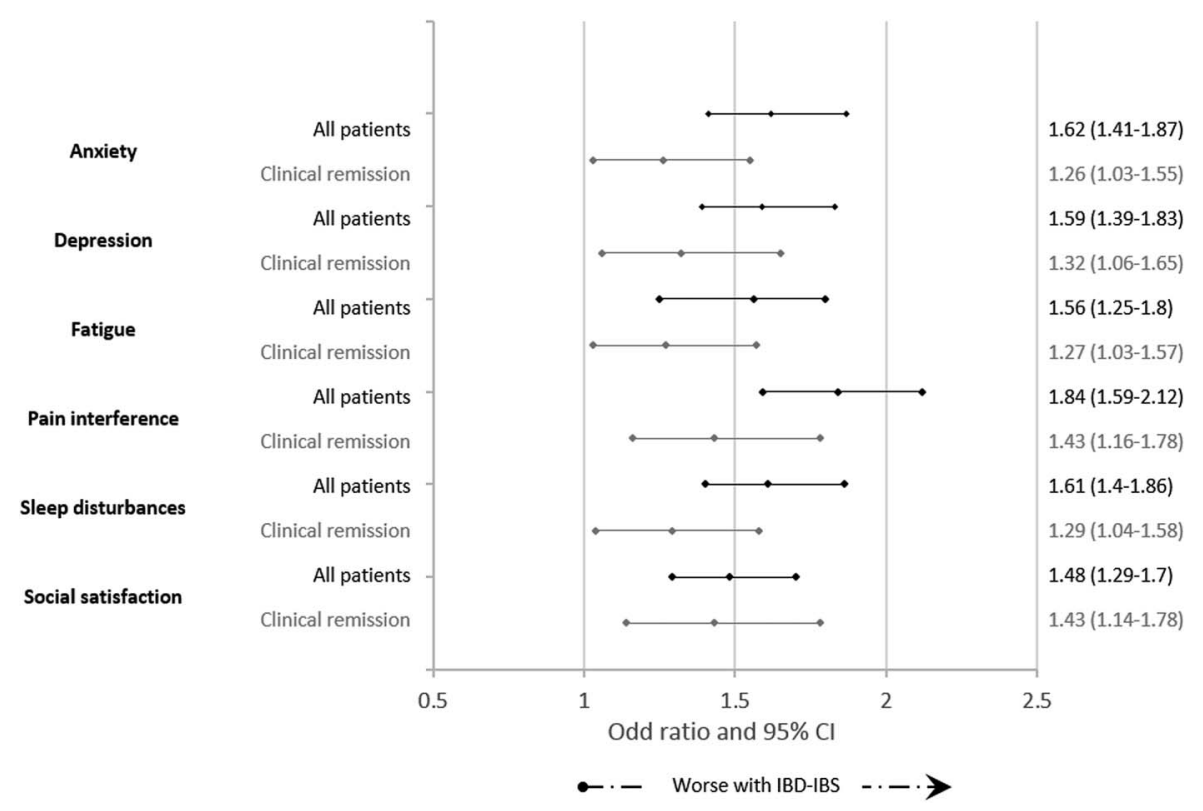

FIGURE 2. Impact of IBD-IBS on PROs. Data represent ORs and $95 \% \mathrm{Cls}$. 
We also recognize a number of limitations to our study. First, all data in our cohort are self-reported with inability to verify selfreported information in each participant. Nevertheless, a recent validation study revealed $97 \%$ accuracy rate of self-reported IBD status and disease subtypes within the CCFA Partners cohort. ${ }^{37}$ Another limitation concerning the self-reported nature of the study is the IBS diagnosis. In our cohort, patients with IBD-IBS were identified on the basis of the participants' report that they were told they had IBS by their treating physician. Hence, the exact IBS criteria used to confirm the diagnosis are unknown. In addition, it is unclear whether other potential causes of the patient's symptoms were ruled out or treated before IBS diagnosis (e.g., small intestinal bacterial overgrowth and celiac disease). Those uncertainties in the diagnosis make this a potentially heterogeneous group of IBD patients with IBS-like symptoms rather than a unified cohort of patients with IBD-IBS. Another limitation to this study is the inability to objectively confirm the presence and severity of active inflammation. This is important as active disease can independently impact the individual patient's quality of life and functional status and may be a source of bias in our analyses. In addition, the clinical measures of disease activity, sCDAI and SCCAI, can be elevated in patients with IBS who have no active inflammation. Hence, using these indices to define clinical disease activity may result in misclassification of some patients with IBD-IBS. Nevertheless, concomitant IBS diagnosis remains a significant predictor for adverse PROs even after excluding patients with significant GI symptoms meeting the clinical criteria of active IBD suggesting that those associations are true.

In conclusion, in this cross-sectional study of patients with IBD, there was a high prevalence of coexisting IBS diagnosis in both patients with $\mathrm{CD}$ and UC/IC. Furthermore, concomitant IBS diagnosis was associated with high rates of narcotic use, increased health resources utilization, lower quality of life, and adverse PROs. As each of these influences can negatively impact an IBD patient's care, it is important to identify patients with a concomitant IBS diagnosis and provide the appropriate counseling and medical therapy.

\section{REFERENCES}

1. Isgar B, Harman M, Kaye MD, et al. Symptoms of irritable bowel syndrome in ulcerative colitis in remission. Gut. 1983;24:190-192.

2. Minderhoud IM, Oldenburg B, Wismeijer JA, et al. IBS-like symptoms in patients with inflammatory bowel disease in remission; relationships with quality of life and coping behavior. Dig Dis Sci. 2004;49:469-474.

3. Ansari R, Attari F, Razjouyan H, et al. Ulcerative colitis and irritable bowel syndrome: relationships with quality of life. Eur J Gastroenterol Hepatol. 2008;20:46-50.

4. Barratt SM, Leeds JS, Robinson K, et al. Reflux and irritable bowel syndrome are negative predictors of quality of life in coeliac disease and inflammatory bowel disease. Eur J Gastroenterol Hepatol. 2011;23: $159-165$.

5. Fukuba N, Ishihara S, Tada $\mathrm{Y}$, et al. Prevalence of irritable bowel syndrome-like symptoms in ulcerative colitis patients with clinical and endoscopic evidence of remission: prospective multicenter study. Scand $J$ Gastroenterol. 2014;49:674-680.

6. Halpin SJ, Ford AC. Prevalence of symptoms meeting criteria for irritable bowel syndrome in inflammatory bowel disease: systematic review and meta-analysis. Am J Gastroenterol. 2012;107:1474-1482.
7. Grover M, Herfarth H, Drossman DA. The functional-organic dichotomy: postinfectious irritable bowel syndrome and inflammatory bowel diseaseirritable bowel syndrome. Clin Gastroenterol Hepatol. 2009;7:48-53.

8. Quigley EM. Overlapping irritable bowel syndrome and inflammatory bowel disease: less to this than meets the eye? Therap Adv Gastroenterol. 2016;9:199-212

9. Ramos-Rivers C, Regueiro M, Vargas EJ, et al. Association between telephone activity and features of patients with inflammatory bowel disease. Clin Gastroenterol Hepatol. 2014;12:986-994. e1.

10. Long MD, Barnes EL, Herfarth $\mathrm{HH}$, et al. Narcotic use for inflammatory bowel disease and risk factors during hospitalization. Inflamm Bowel Dis. 2012;18:869-876.

11. Kappelman MD, Long MD, Martin C, et al. Evaluation of the patientreported outcomes measurement information system in a large cohort of patients with inflammatory bowel diseases. Clin Gastroenterol Hepatol. 2014;12:1315-1323. e2.

12. Chey WD, Kurlander J, Eswaran S. Irritable bowel syndrome: a clinical review. JAMA. 2015;313:949-958.

13. Simren M, Axelsson J, Gillberg R, et al. Quality of life in inflammatory bowel disease in remission: the impact of IBS-like symptoms and associated psychological factors. Am J Gastroenterol. 2002;97:389-396.

14. Keohane J, O'Mahony C, O'Mahony L, et al. Irritable bowel syndrometype symptoms in patients with inflammatory bowel disease: a real association or reflection of occult inflammation? Am J Gastroenterol. 2010; 105:1789-1794. quiz 1795.

15. Piche T, Ducrotte P, Sabate JM, et al. Impact of functional bowel symptoms on quality of life and fatigue in quiescent crohn disease and irritable bowel syndrome. Neurogastroenterol Motil. 2010;22:626-e174.

16. Jelsness-Jorgensen LP, Bernklev T, Moum B. Fatigue and disease-related worries among inflammatory bowel disease patients in remission; is it a reflection of coexisting IBS-like symptoms? A short report. $J$ Psychosom Res. 2012;73:469-472.

17. Berrill JW, Gallacher J, Hood K, et al. An observational study of cognitive function in patients with irritable bowel syndrome and inflammatory bowel disease. Neurogastroenterol Motil. 2013;25:918-e704.

18. Jonefjall B, Strid H, Ohman L, et al. Characterization of IBS-like symptoms in patients with ulcerative colitis in clinical remission. Neurogastroenterol Motil. 2013;25:756-e578.

19. Vivinus-Nebot M, Frin-Mathy G, Bzioueche H, et al. Functional bowel symptoms in quiescent inflammatory bowel diseases: role of epithelial barrier disruption and low-grade inflammation. Gut. 2014;63:744-752.

20. Long MD, Kappelman MD, Martin CF, et al. Development of an internetbased cohort of patients with inflammatory bowel diseases (CCFA partners): methodology and initial results. Inflamm Bowel Dis. 2012;18:2099-2106.

21. Rothrock NE, Hays RD, Spritzer K, et al. Relative to the general US population, chronic diseases are associated with poorer health-related quality of life as measured by the patient-reported outcomes measurement information system (PROMIS). J Clin Epidemiol. 2010;63:1195-1204.

22. Cella D, Yount S, Rothrock N, et al. The patient-reported outcomes measurement information system (PROMIS): progress of an NIH roadmap cooperative group during its first two years. Med Care. 2007;45(5 suppl 1):S3-S11.

23. Yost KJ, Eton DT, Garcia SF, et al. Minimally important differences were estimated for six patient-reported outcomes measurement information system-cancer scales in advanced-stage cancer patients. J Clin Epidemiol. 2011;64:507-516.

24. Swanholm E, McDonald W, Makris U, et al. Estimates of minimally important differences (MIDs) for two patient-reported outcomes measurement information system (PROMIS) computer-adaptive tests in chronic pain patients. $J$ Appl Biobehavioral Res. 2014;19:217-232.

25. Thissen D, Liu Y, Magnus B, et al. Estimating minimally important difference (MID) in PROMIS pediatric measures using the scalejudgment method. Qual Life Res. 2016;25:13-23.

26. Farrokhyar F, Marshall JK, Easterbrook B, et al. Functional gastrointestinal disorders and mood disorders in patients with inactive inflammatory bowel disease: prevalence and impact on health. Inflamm Bowel Dis. 2006;12:38-46.

27. Kim ES, Cho KB, Park KS, et al. Predictive factors of impaired quality of life in korean patients with inactive inflammatory bowel disease: association with functional gastrointestinal disorders and mood disorders. J Clin Gastroenterol. 2013;47:e38-e44. 
28. Agarwal N, Spiegel BM. The effect of irritable bowel syndrome on healthrelated quality of life and health care expenditures. Gastroenterol Clin North Am. 2011;40:11-19.

29. Ritter PL, Stewart AL, Kaymaz H, et al. Self-reports of health care utilization compared to provider records. J Clin Epidemiol. 2001;54:136-141.

30. Roberts RO, Bergstralh EJ, Schmidt L, et al. Comparison of self-reported and medical record health care utilization measures. J Clin Epidemiol. 1996;49:989-995.

31. Hirsch A, Yarur AJ, Dezheng H, et al. Penetrating disease, narcotic use, and loop ostomy are associated with ostomy and IBD-related complications after ostomy surgery in crohn's disease patients. $J$ Gastrointest Surg. 2015;19:1852-1861.

32. Lichtenstein GR, Feagan BG, Cohen RD, et al. Serious infections and mortality in association with therapies for crohn's disease: TREAT registry. Clin Gastroenterol Hepatol. 2006;4:621-630.
33. Targownik LE, Nugent $Z$, Singh $H$, et al. The prevalence and predictors of opioid use in inflammatory bowel disease: a population-based analysis. Am J Gastroenterol. 2014;109:1613-1620.

34. Cross RK, Wilson KT, Binion DG. Narcotic use in patients with crohn's disease. Am J Gastroenterol. 2005;100:2225-2229.

35. Edwards JT, Radford-Smith GL, Florin TH. Chronic narcotic use in inflammatory bowel disease patients: prevalence and clinical characteristics. J Gastroenterol Hepatol. 2001;16:1235-1238.

36. Hanson KA, Loftus EV Jr, Harmsen WS, et al. Clinical features and outcome of patients with inflammatory bowel disease who use narcotics: a case-control study. Inflamm Bowel Dis. 2009;15: 772-777.

37. Randell RL, Long MD, Cook SF, et al. Validation of an internet-based cohort of inflammatory bowel disease (CCFA partners). Inflamm Bowel Dis. 2014;20:541-544. 